\title{
Une « Mallette Scan Champ Proche » pour l'enseignement de la compatibilité électromagnétique
}

\author{
T. Dubois, J-P. Guillet, G. Duchamp, J. Tomas \\ IMS et pôle CNFM de Bordeaux, Université de Bordeaux, Talence, France \\ Contact email : tristan.dubois@u-bordeaux.fr
}

\begin{abstract}
Avec l'augmentation du nombre de systèmes électroniques, les dysfonctionnements causés par des problèmes de compatibilité électromagnétique (CEM) sont de plus en plus présents. La formation des futurs ingénieurs dans ce domaine est donc très importante. Cependant, l'enseignement de la CEM n'est pas une tâche facile du fait qu'il faut faire intervenir des notions de rayonnement électromagnétique, de propagation d'onde électromagnétique, de couplage électromagnétique... Un travail important doit donc être réalisé afin de mettre en place des bancs de mesure pédagogiques permettant d'illustrer les phénomènes rencontrés. Dans le domaine de la compatibilité électromagnétique, il existe un banc de mesure nommé «scanner champ proche » qui permet de réaliser des cartographies des rayonnements électromagnétiques en zone de champ proche d'un composant ou d'un circuit électronique. Ce papier présente la conception d'une «Mallette Scan Champ Proche» transportable et autonome permettant la réalisation rapide de cartographies de champ électromagnétique en zone de champ proche. Une vidéo de démonstration est proposée en partie III.
\end{abstract}

Mots clés : CEM, Rayonnement électromagnétique, Champ proche électrique et magnétique, Outil pédagogique.

\section{Introduction}

Depuis plusieurs années, le domaine de la compatibilité électromagnétique (CEM) n'a pas arrêté de prendre de l'ampleur. Cette tendance est principalement explicable par l'augmentation du nombre d'équipements électroniques, l'augmentation des fréquences de fonctionnement, la réduction des tensions d'alimentation induisant une réduction des seuils de compatibilité électromagnétique et l'intégration de plus en plus poussée des circuits électroniques. L'environnement électromagnétique devenant de plus en plus sévère et les circuits électroniques devenant de plus en plus sensibles, il est indispensable de former les futurs ingénieurs au domaine de la CEM.

La compréhension des phénomènes liés au domaine de la compatibilité électromagnétique n'est pas simple. En effet, la plupart des phénomènes mettent en jeu les ondes électromagnétiques. Aussi il faut être capable, par exemple, de comprendre les phénomènes propres à l'émission des ondes électromagnétiques, c'est-à-dire les phénomènes de résonance, d'onde stationnaire, de propagation et de couplage électromagnétique. Tous ces phénomènes classiquement introduits dans les enseignements d'électromagnétisme ou d'hyperfréquence sont difficiles à conceptualiser par les étudiants du fait qu'ils sont pour la plupart très abstraits. Il est donc important de développer des 
bancs de mesure ou d'expérimentation pouvant être utilisés pendant les cours et permettant d'illustrer via la mesure ces phénomènes électromagnétiques.

Dans le domaine de la compatibilité électromagnétique, il existe un banc de mesure nommé scanner champ proche (NFS - Near Field Scanner) qui permet de réaliser des cartographies des rayonnements électromagnétiques émis par un composant ou un circuit électronique en zone de champ proche. Ce banc utilisé dans les domaines de la recherche et industriel permet ainsi d'analyser le rayonnement de dispositifs tels que des cartes électroniques mais aussi d'un composant électronique [1][2][3]. Ce type de banc bien que très utile d'un point de vu pédagogique reste cependant cher et difficilement transportable.

Le but de cet article est donc de présenter un banc de mesure électromagnétique portable permettant la réalisation de cartographies des champs électriques et magnétiques rayonnés en zone de champ proche par un composant ou un circuit électronique.

La première partie du papier présente le projet dans sa globalité ainsi que le concept de «mallette scan champ proche ». La deuxième partie concerne la présentation des différentes parties constituant la mallette. Précisons que toutes les différentes parties du banc ont été réalisées par des étudiants de niveau Licence 2, 3 et Master 1, lors de projets et de stages de courtes durées. La troisième partie est consacrée à la présentation de différents résultats d'étude ayant pour but d'illustrer les possibilités de la «mallette scan champ proche ».

\section{Le projet « Mallette Scan Champ Proche »}

$\underline{\text { But et description du projet }}$

Ce projet pédagogique consiste à développer un banc de mesure permettant la caractérisation des champs électromagnétiques rayonnés en zone de champ proche par des circuits et cartes électroniques, comme décrit par la norme IEC-61967-3. Pédagogiquement, ce projet a deux objectifs principaux. Le premier est d'utiliser ce banc de mesure lors de cours magistraux, de travaux dirigés et de travaux pratiques afin d'illustrer des phénomènes physiques faisant intervenir des rayonnements électromagnétiques. Sa conception doit alors le rendre facilement transportable, autonome énergétiquement, ludique et peu expansif. Le deuxième objectif est de faire réaliser cette mallette par les étudiants de niveau allant de la Licence 2 jusqu'en Master 1 dans le cadre de projets et de stages de courtes durées traitant des différents domaines de l'EEA.

Le cahier des charges est donc simple. Le banc de mesure doit être réalisé par des étudiants, doit être autonome et doit tenir dans une valise adaptée à son utilisation, afin qu'il puisse être transportable pour être utilisé dans des enseignements.

Le concept de la «mallette scan champ proche »: une mallette faite par les étudiants pour les enseignants

L'idée de la «mallette scan champ proche » est partie d'un banc de mesure existant dans le domaine de la recherche et de l'industrie en compatibilité électromagnétique. Un scan champ proche est un banc de mesure permettant de réaliser des cartographies en zone de champ proche des champs électrique et magnétique émis par un dispositif. Il est principalement construit autour de deux éléments primordiaux à son fonctionnement : une table de déplacement composée de moteurs et d'une sonde de champ proche [1][2][3]. La réalisation d'une cartographie consiste à balayer (ou scanner) la surface d'un circuit sous test et de relever en tout point, à travers la sonde de champ proche, un signal électrique image du champ rayonné par le circuit. Le schéma de la figure 1 présente le principe de base d'un scan champ proche et la réalisation d'une cartographie. 

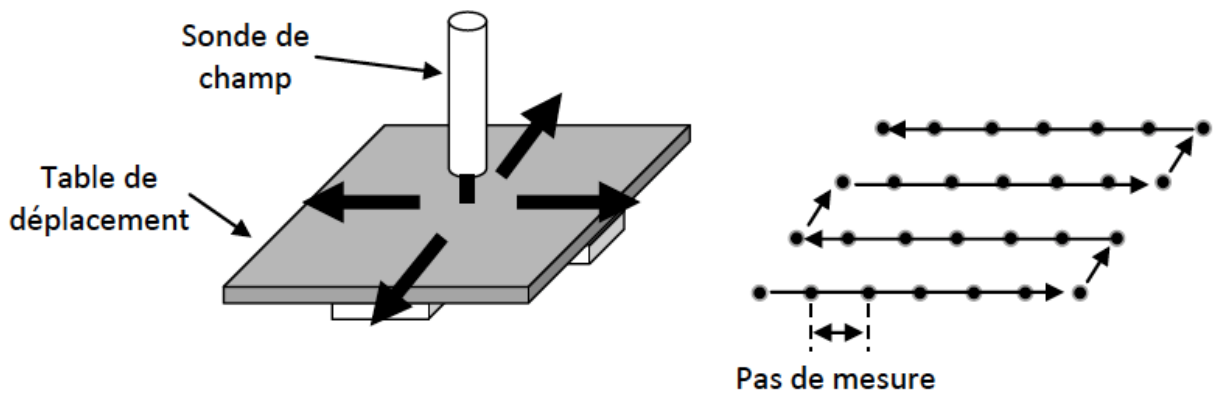

Fig. 1. Principe d'une cartographie.

La «Mallette Scan Champ Proche » a donc été construite autour de ces deux éléments. Le travail de conception réalisé a consisté à définir les éléments nécessaires au bon fonctionnement du système de déplacement et de détection mais permettant également de rendre tout le banc de mesure autonome et transportable afin qu'il soit facilement utilisable dans une salle de cours. La figure 2 présente la structure de la «Mallette Scan Champ Proche ».

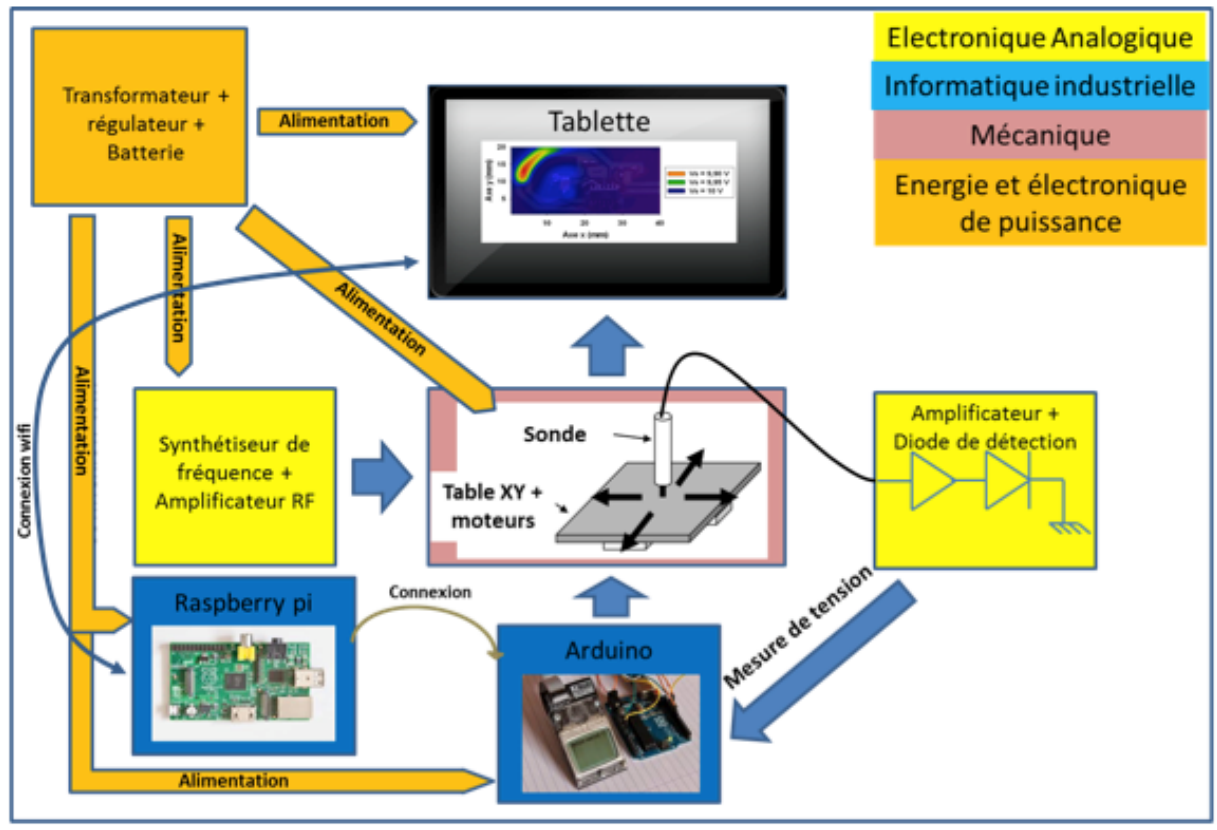

Fig. 2. Schéma de la «Mallette Scan Champ Proche».

La «Mallette Scan Champ Proche » que nous appellerons NFSC pour Near Field Scanner Case est donc constituée de plusieurs éléments permettant de répondre au cahier des charges défini précédemment. Le projet de conception de la NFSC a donc été découpé en quatre tâches décrites ci-dessous :

Tâche 1 : Électronique de puissance

Elle concerne la réalisation du circuit d'alimentation dont le rôle est d'amener, à partir de la tension d'une batterie, toutes les tensions nécessaires aux différents éléments de la mallette en respectant bien évidemment les différents courants de consommation. 
Tâche 2 : Hyperfréquence

Elle concerne la caractérisation et l'assemblage des différents modules hyperfréquences de la mallette. Cela concerne les oscillateurs contrôlés en tension (VCOs) qui doivent alimenter les lignes micro-rubans ce qui permet, notamment, d'illustrer des phénomènes d'ondes stationnaires, le circuit de détection construit autour d'une diode de détection ainsi que les sondes de champ proche. Cette tâche concerne aussi la détermination du coefficient de calibrage des sondes permettant de déterminer la valeur réelle du champ mesuré.

Tâche 3 : Mécanique

Elle concerne principalement l'assemblage de la table XY et la réalisation de la mallette. Elle consiste aussi à s'assurer de l'ergonomie du banc de mesure et son intégration dans la mallette.

Tâche 4 : Informatique industriel

Elle concerne la configuration et la programmation du Raspberry-pi. Ce dernier a pour rôle premièrement de contrôler les moteurs pour la réalisation de la cartographie via une carte Arduino, deuxièmement d'effectuer la mesure de tension en sortie de sonde via cette même carte Arduino, troisièmement de contrôler les VCOs, et quatrièmement de communiquer avec une tablette PC ou un smartphone afin de visualiser le résultat de la cartographie.

\section{III.Conception et Test}

Dans cette section, nous présentons le banc de mesure qui a été fabriqué en expliquant le fonctionnement des différents éléments du banc ainsi que les différentes études réalisées dans le cadre des différentes tâches détaillées dans la section précédente. La figure 3 présente une photo du banc de mesure réalisé. Une vidéo de la mesure de la composante verticale du champ électrique rayonné par une ligne micro-ruban est aussi disponible via le lien suivant: Mesure_du_champ_Ez_Rayonne_par_une _Ligne_Microruban.mp4

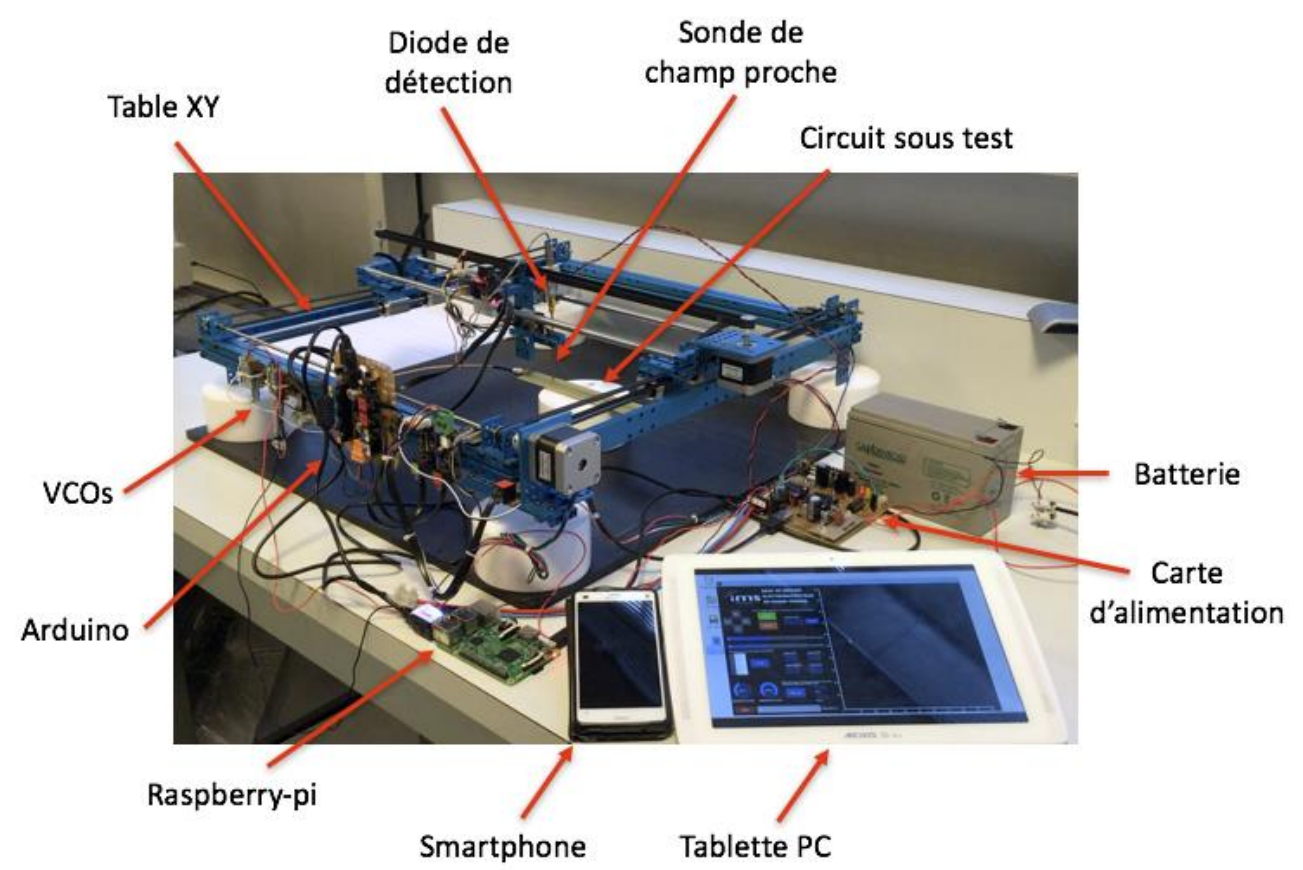

Fig. 3. Photo du banc de mesure réalisé par les étudiants avant incorporation dans la mallette. 


\section{$\underline{\text { La table XY et l'Arduino }}$}

La table XY a été réalisée à partir d'une table traçante « Plotter XY »V2.0 de la société Makeblock. A l'origine, cette table est conçue pour réaliser des dessins très complexes en Gcode. Elle se compose d'une structure en aluminium anodisé associée à 2 moteurs pas-àpas et à une électronique de puissance supervisée par une platine compatible Arduino. Afin d'utiliser cette table pour la réalisation de cartographie champ proche, le stylo a été remplacé par une sonde de champ proche associée au système de détection. Une grande partie du travail a consisté à intégrer la sonde à la table. Le système de détection fournit à l'Arduino une tension continue représentant l'image du rayonnement capté par la sonde. Il a ensuite fallu développer le programme pour l'Arduino permettant de réaliser la cartographie. Cela consiste donc à suivre la procédure décrite en Figure 1, c'est-à-dire effectuer un balayage de la sonde au-dessus du circuit sous test et de mesurer en chaque point la tension continue envoyée par le système de détection. Cette tension continue est ensuite utilisée pour calculer le champ électrique ou magnétique détecté par la sonde. $\mathrm{Au}-$ delà de ces tâches, l'Arduino est aussi utilisé pour régler la fréquence de sortie de deux VCOs via une sortie délivrant des PWM (Pulse Width Modulation) filtrés afin d'obtenir des tensions continues.

\section{Les sondes de champ proche}

La sonde de champ proche est un des éléments les plus importants du banc de mesure. C'est elle qui permet la détection des différentes composantes du champ électrique et magnétique. Les sondes de champ proche sont réalisées à partir d'un câble coaxial semirigide présentant une impédance caractéristique de $50 \Omega$. L'âme centrale du câble est dénudée. Suivant la forme que l'on donne à l'âme centrale, il est alors possible de détecter les différentes composantes du champ électrique ou magnétique rayonnées par un dispositif. La figure 4 présente deux types de sondes de champ proche.

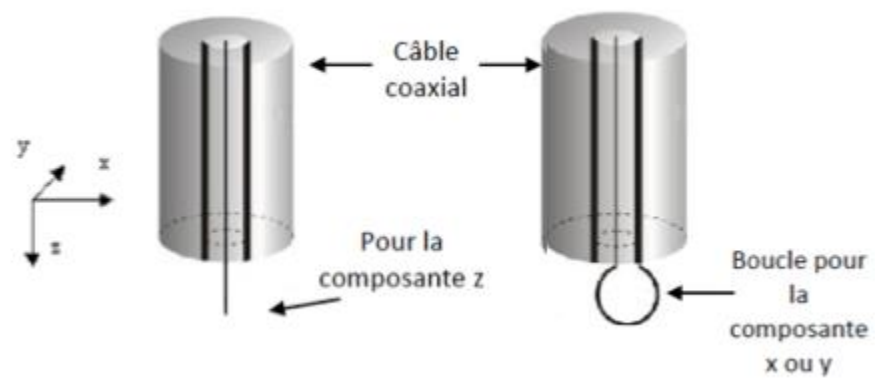

(a)

(b)

Fig. 4. Sondes de champ proche permettant la détection de la composant (a) $\mathrm{z}$ du champ électrique et (b) $\mathrm{x}$ ou y de champ magnétique.

Davantage d'informations sur les sondes de champ proche peuvent être trouvées dans les références [1][4][5]. Le travail produit a été principalement la réalisation des sondes.

\section{Système de détection et calibration}

Le système de détection, connecté à la sonde de champ proche, est principalement composé d'une diode de détection encapsulée de la société Mini-Circuits qui permet de convertir un signal sinusoïdal de fréquence allant jusqu'à $18 \mathrm{GHz}$ et de puissance pouvant 
varier entre $-60 \mathrm{dBm}$ et $0 \mathrm{dBm}$, en une tension continue. La figure 5 présente une photo de la diode Schottky de détection associée à la sonde de champ proche.

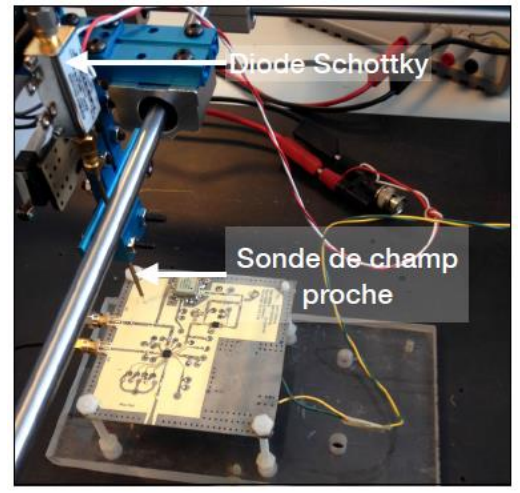

Fig. 5. Photo de la diode de détection associée à la sonde de champ proche.

Un des buts du banc de mesure est de restituer la valeur du champ électrique ou magnétique par la sonde de champ proche. Dans sa configuration actuelle, le signal sinusoïdal image du champ capté par la sonde est converti en une tension continue par la diode de détection. Afin de remonter à la valeur du champ électrique ou magnétique, une phase de calibration est nécessaire. La phase de calibration consiste premièrement à déterminer une relation entre l'amplitude $\mathrm{V}_{\text {in }}$ de la tension alternative captée par la sonde et la tension continue de sortie $\mathrm{V}_{\text {out }}$ de la diode de détection. La deuxième étape consiste à déterminer un coefficient de calibration permettant de relier le champ électrique ou magnétique à l'amplitude $\mathrm{V}_{\text {in }}$.

La relation entre l'amplitude $\mathrm{V}_{\text {in }}$ et la puissance $\mathrm{P}_{\text {in }}$ du signal à l'intérieur de la sonde est donnée en équation (1).

$$
V_{\text {in }}=\sqrt{P_{\text {in }} * Z_{C}}
$$

$\mathrm{Z}_{\mathrm{c}}$ correspond à l'impédance d'entrée de la diode de détection.

La figure 6 représente la mesure de la tension continue de sortie de la diode de détection en fonction de la puissance du signal d'entrée $\mathrm{P}_{\text {in }}$ exprimée en $\mathrm{dBm}$. Ce résultat a été obtenu en mesurant la composante verticale du champ électrique rayonné par une ligne micro-ruban.

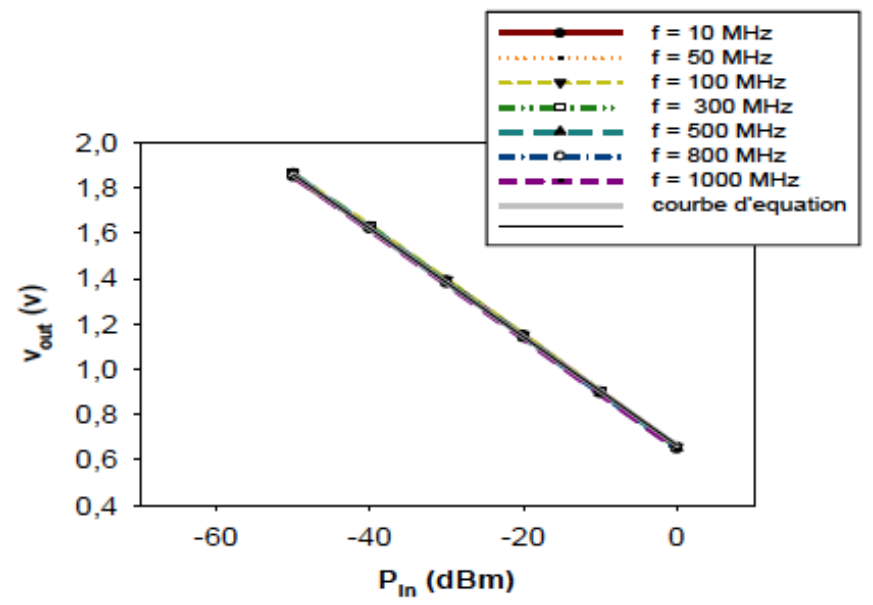

Fig. 6. Tension continue $\mathrm{V}_{\text {out }}$ de sortie de la diode de détection en fonction de la puissance $\mathrm{P}_{\text {in }}$ du signal d'entrée de la sonde de champ proche. 
La variation observée sur la figure 6 peut être modélisée par une droite dont l'équation est donnée en (2).

$$
V_{\text {out }}=-0,239 P_{\text {in }}+0,6649
$$

En utilisant (1) et (2), il est alors possible de définir une relation entre l'amplitude de la tension Vin image du champ capté par la sonde et la tension continue $\mathrm{V}_{\text {out }}$ de sortie de la diode de détection.

La détermination du coefficient de calibration permettant d'exprimer la valeur du champ électrique ou magnétique capté par la sonde en fonction de l'amplitude du signal mesuré par la sonde se fait classiquement en comparant des résultats de mesure et de simulation électromagnétique 3D réalisés sur un dispositif bien connu comme par exemple une ligne micro-ruban présentant une impédance caractéristique de 50 Ohms. La figure 7 illustre la mesure réalisée et le modèle de simulation associé, réalisé sur le logiciel HFSS.

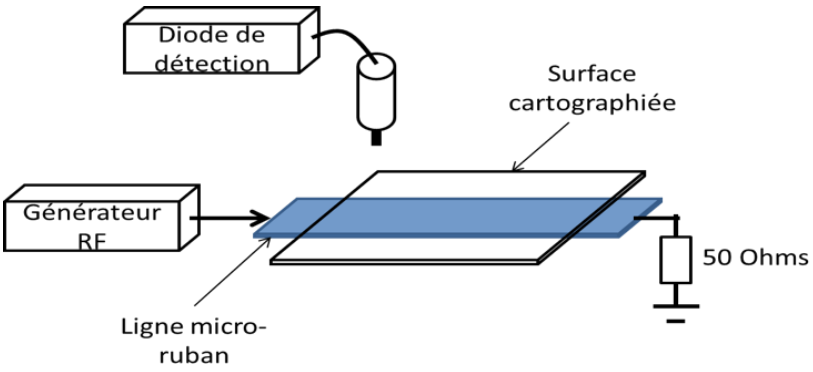

(a)

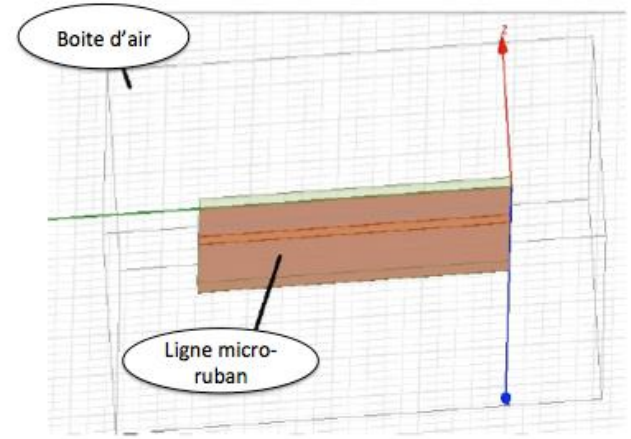

(b)

Fig. 7. (a) Configuration de mesure et (b) modèle de simulation utilisé pour la détermination du coefficient de calibrage de la sonde électrique $E_{z}$.

La figure 8 présente le résultat de la cartographie obtenue représentant la mesure de la composante verticale du champ électrique rayonnée à $1 \mathrm{~mm}$ au-dessus de la ligne microruban. L'amplitude de la mesure correspond à l'amplitude Vin de la tension présent dans la sonde pour une fréquence d'excitation de la ligne micro-ruban de $500 \mathrm{MHz}$ et d'une tension d'excitation de $1 \mathrm{~V}$.

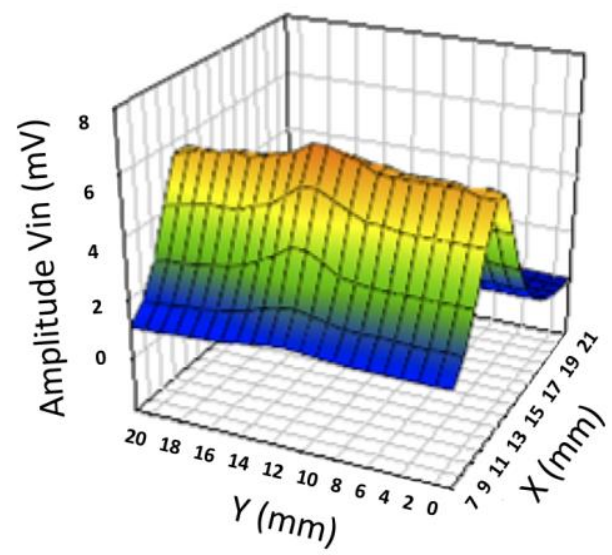

Fig. 8. Cartographie de la composante verticale du champ électrique à $1 \mathrm{~mm}$ au-dessus de la ligne microruban réalisée pour une fréquence d'excitation de la ligne de $50 \mathrm{MHz}$. 
La même étude est réalisée en simulation à la différence près que l'amplitude mesurée correspond à la valeur de la composante verticale du champ électrique $E_{z}$ en $(\mathrm{V} / \mathrm{m})$ rayonnée par la ligne à $1 \mathrm{~mm}$.

En comparant les résultats de mesure et de simulation, il est possible de montrer que l'amplitude Vin et le champ électrique $\mathrm{E}_{\mathrm{z}}$ sont proportionnels et peuvent donc être liées par la relation (3).

$$
E_{Z}=K(f) * V_{\text {in }}
$$

K représente le coefficient de calibration de la sonde.

La figure 9 présente l'amplitude $\mathrm{V}_{\text {in }}$ et $\mathrm{E}_{\mathrm{z}}$ respectivement de la tension dans la sonde obtenue par mesure et de la composante verticale du champ électrique rayonnée par la ligne micro-ruban obtenue en simulation en fonction de l'amplitude de la tension $\mathrm{V}_{\mathrm{e}} \mathrm{d}$ 'excitation de la ligne micro-ruban pour une fréquence d'excitation de $500 \mathrm{MHz}$.

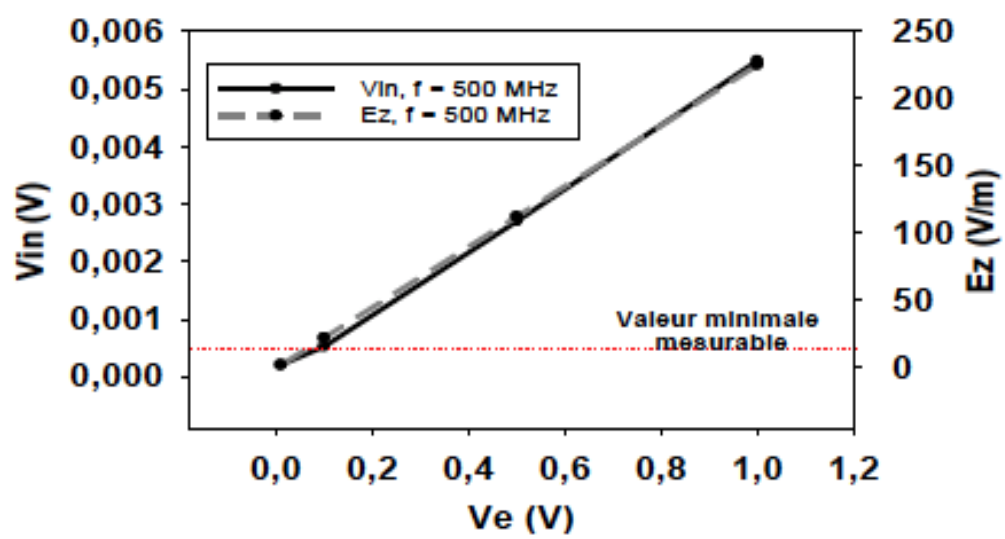

Fig. 9. Amplitudes $V_{\text {in }}$ et $E_{z}$ respectivement de la tension dans la sonde obtenue par mesure et de la composante verticale du champ électrique obtenue par simulation rayonnée par la ligne micro-ruban en fonction de l'amplitude de la tension $\mathrm{V}_{\mathrm{e}} \mathrm{d}$ 'excitation de la ligne micro-ruban pour une fréquence d'excitation de $500 \mathrm{MHz}$.

Le coefficient $\mathrm{K}$ en fonction de la fréquence est donné en figure 10.

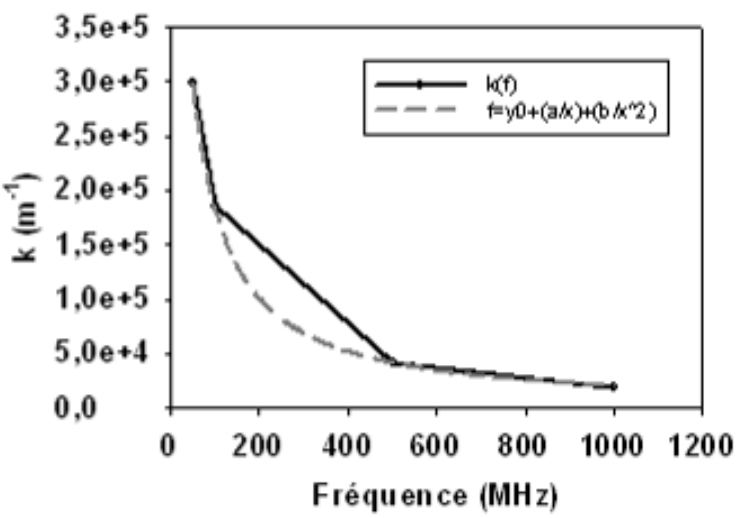

Fig. 10. Coefficient $\mathrm{K}$ en fonction de la fréquence

D’après la figure 10 le coefficient $\mathrm{K}(\mathrm{f})$ peut être modélisé par une relation de la forme :

$$
K=y_{0}+\frac{a}{f}+\frac{b}{f^{2}} \text {. }
$$


Configuration du Raspberry-pi et du logiciel pour le pilotage de l'Arduino et de la communication avec la tablette PC

La NFSC sera principalement utilisée lors de démonstrations faites en cours et en TD ou lors de TP. Aussi, il est important d'une part que la mallette puisse être transportable facilement mais aussi que la visualisation des résultats de cartographie soit facile et ludique via l'utilisation de tablettes ou smartphones par exemple. La figure 11 présente le concept de la communication sans fils de la mallette.

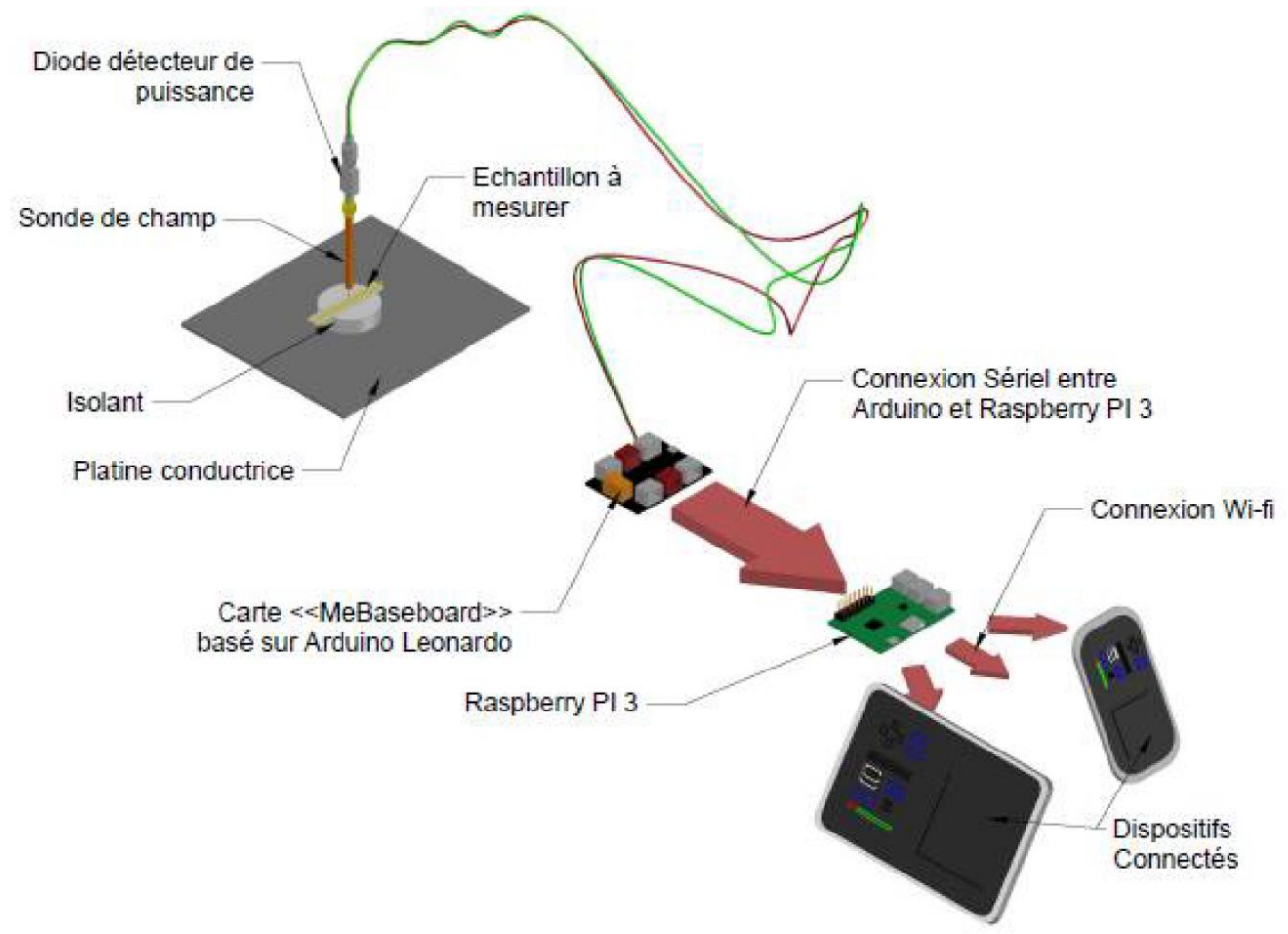

Fig. 11. Concept de la communication sans fils de la mallette

Le cahier des charges de cette tâche est le suivant :

- Construire une interface permettant de lancer le processus de cartographie (placement de la sonde, réglage de la dimension de la cartographie, visualisation du résultat et enregistrement des données),

- Construire une page web accessible via une tablette ou un smart phone afin que les étudiants puissent visualiser les résultats de la cartographie sur leur propre appareil.

Pour le premier point, la solution retenue a été de se connecter au Rasperry-pi en utilisant l'outil VCN (Virtual Network Computing). Cet outil permet de prendre le contrôle du Rasperry-pi via une tablette, un ordinateur ou un smart phone, et de lancer une application java script. L'outil Processing a ensuite été utilisé afin de créer un logiciel permettant de communiquer avec l'Arduino (contenant le programme de lancement de la cartographie et la lecture de la tension de sortie de la diode de détection) et d'afficher le résultat de la cartographie. Un des avantages de l'outil Processing en plus d'être gratuit et qu'il est disponible sur les différentes plateformes telles que Windows, Mac OS ou Linux. Le logiciel développé est présenté sur la figure 12 (a). 
Pour le deuxième point, la solution retenue est la suivante : une fois la cartographie terminée, un bouton web présent sur le logiciel permet de lancer un script mathematica permettant de générer un graphique $2 \mathrm{D}$ et $3 \mathrm{D}$, et parallèlement d'ouvrir une page web permettant de visualiser les résultats de la cartographie réalisée. La figure 12 (b) présente la page web et un exemple de cartographie.
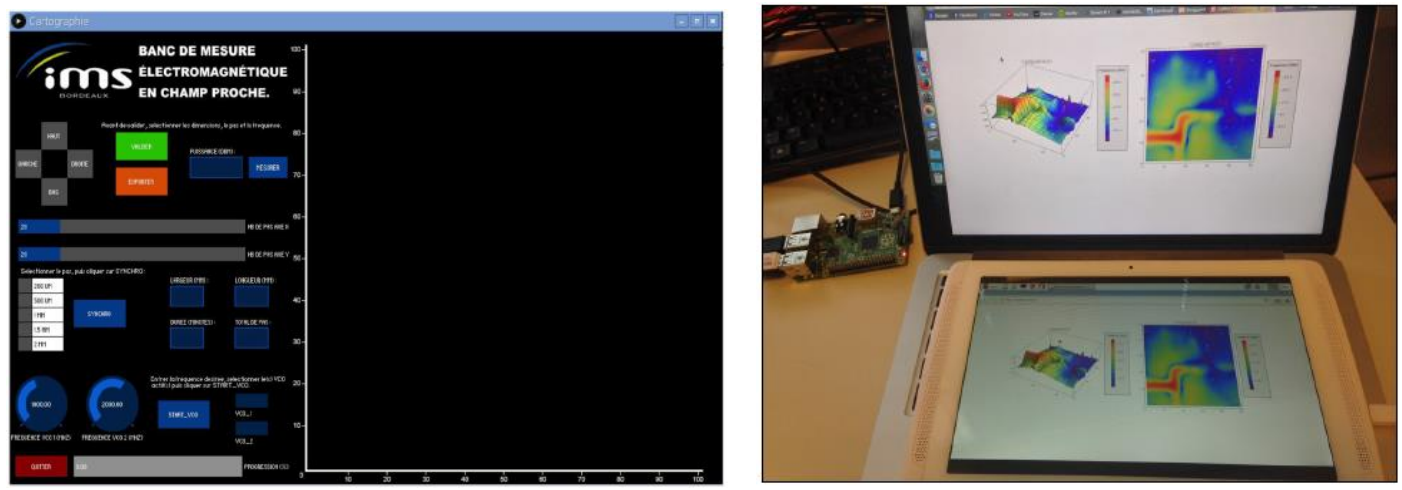

Fig. 12. (a) Logiciel développé avec Processing permettant le réglage de la dimension et du pas de la cartographie, l'activation du VCO permettant l'excitation du dispositif sous test, le lancement de la cartographie ainsi que sa visualisation, (b) Visualisation des résultats de cartographie via la page web.

\section{Carte d'alimentation}

La carte d'alimentation a pour rôle de fournir les différentes tensions nécessaires à l'alimentation de tous les éléments de la NFSC à partir d'une batterie $12 \mathrm{~V}$. Le travail a donc consisté à réaliser la carte, la tester puis déterminer l'autonomie de la batterie lorsqu'une cartographie est lancée. Le tableau 1 récapitule les tensions, courants, puissances et énergies des différents éléments du banc de mesure permettant de définir le cahier des charges de la carte d'alimentation.

TABLEAU I. Tensions, courants, puissances et énergies des différents éléments du banc de mesure permettant de définir le cahier des charges de la carte d'alimentation.

\begin{tabular}{|c|c|c|c|c|c|c|}
\hline & Batterie & $\begin{array}{c}\text { Rapberry- } \\
\text { pi }\end{array}$ & $\begin{array}{l}\text { Arduino } \\
\text { (Carte } \\
\text { principale) }\end{array}$ & $\begin{array}{c}\text { Arduino } \\
\text { (Cartes } \\
\text { moteurs) }\end{array}$ & $\begin{array}{l}\text { Diode de } \\
\text { détection }\end{array}$ & VCOs \\
\hline $\begin{array}{l}\text { Tension } \\
\text { d'alimentation }\end{array}$ & $12 \mathrm{~V}$ & $5 \mathrm{~V}$ & \multirow{4}{*}{$\begin{array}{c}\text { Alimenté } \\
\text { à travers } \\
\text { la carte } \\
\text { Rasperry- } \\
\text { pi }\end{array}$} & $12 \mathrm{~V}$ & $5 \mathrm{~V}$ & $5 \mathrm{~V}$ \\
\hline $\begin{array}{l}\text { Courant } \\
\text { maximum }\end{array}$ & & $0,6 \mathrm{~A}$ & & $3 \mathrm{~A}$ & $0,12 \mathrm{~A}$ & $0,05 \mathrm{~A}$ \\
\hline $\begin{array}{l}\text { Puissance } \\
\text { maximum }\end{array}$ & & $3 \mathrm{~W}$ & & $36 \mathrm{~W}$ & $0,6 \mathrm{~W}$ & $0,25 \mathrm{~W}$ \\
\hline Energie & $302,4 \mathrm{~kJ}$ & & & & & \\
\hline
\end{tabular}

La carte d'alimentation est construite autour de deux convertisseurs Flyback de la société Linear Technology permettant à partir d'une plage de tension autour de $12 \mathrm{~V}$ de délivrer des tensions stables de $12 \mathrm{~V}$ et $5 \mathrm{~V}$ et de fournir les courants nécessaires au fonctionnement des différents éléments de la mallette. La carte d'alimentation est aussi équipée d'un fusible de protection et d'un indicateur de niveau de charge de la batterie. La figure 13 (a) présente une photo de la carte d'alimentation. 
Le test de la carte d'alimentation consiste à vérifier sa capacité à fournir les différents tensions et courants nécessaires au fonctionnement des différents éléments de la mallette. Dans un premier temps, les différents éléments de la mallette ont été remplacés par des résistances dont les valeurs ont été déterminées pour une consommation précise. La figure 13 (b) présente le schéma de mesure. Etant donnés les courants débités, des rhéostats permettant de supporter des intensités « importantes » ont été utilisés.

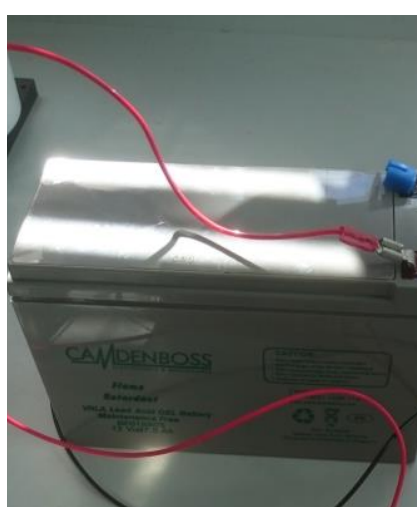

(a)

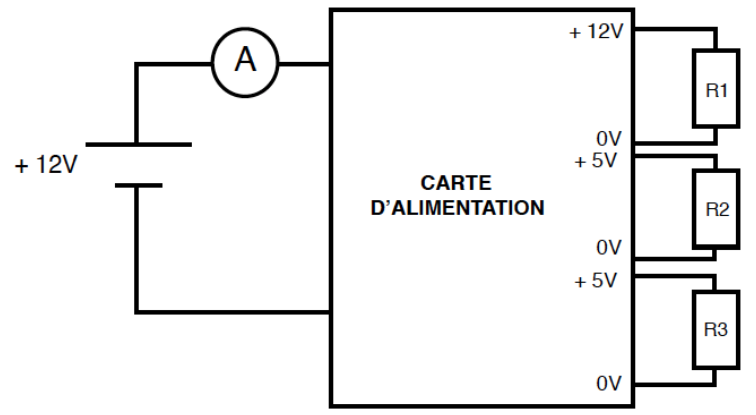

(b)

Fig. 13. (a) Photo de la carte d'alimentation, (b) Schéma permettant de tester la carte d'alimentation.

La valeur des résistances choisies est répertoriée dans le tableau 2.

TABLEAU II. Récapitulatif des courants, tensions et valeurs de résistance choisis pour le test de la carte d'alimentation..

\begin{tabular}{|l|c|c|c|}
\hline & Courant maximal & Tension & $\begin{array}{c}\text { Résistance } \\
\text { équivalente }\end{array}$ \\
\hline Arduino + moteur & $3 \mathrm{~A}$ & $12 \mathrm{~V}$ & $\mathrm{R} 1=4 \Omega$ \\
\hline Diode de détection & $0,12 \mathrm{~A}$ & $5 \mathrm{~V}$ & $\mathrm{R} 2=42 \Omega$ \\
\hline Raspberry-pi & $0,6 \mathrm{~A}$ & $5 \mathrm{~V}$ & $\mathrm{R} 3=8,5 \Omega$ \\
\hline
\end{tabular}

En connectant la batterie à l'entrée de la carte d'alimentation, des mesures de décharges pour différentes consommations ont été réalisées et sont présentées sur la figure 14 .

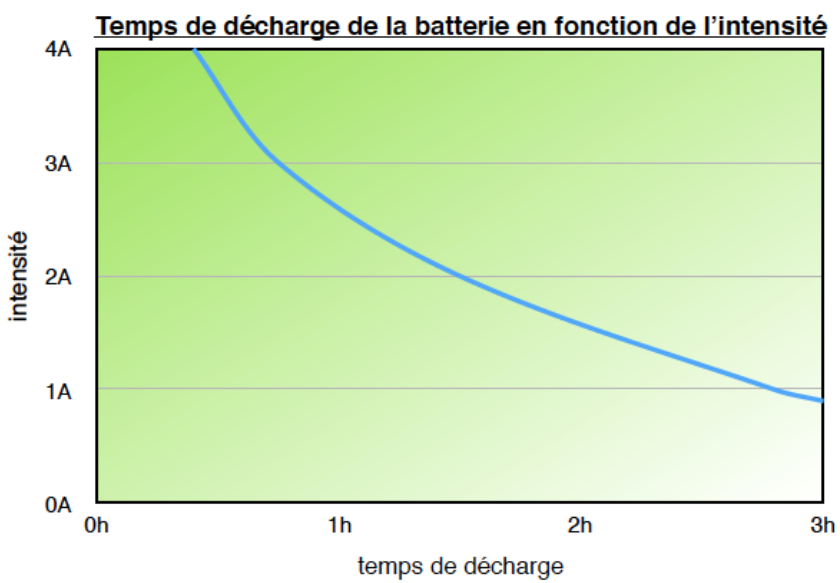

Fig. 14. Courbe de décharge de la batterie en fonction de la consommation de courant. 
D'après la courbe de la figure 14, il apparaît que la batterie peut fournir l'énergie nécessaire à un fonctionnement maximal de la mallette pendant $30 \mathrm{~min}$.

Une fois le banc de mesure connecté à la carte d'alimentation, il a pu être montré qu'en fonctionnement normal la mallette consommait un courant global de l'ordre de 1 A soit une autonomie de presque $3 \mathrm{~h}$ ce qui est largement suffisant pour l'application pédagogique visée.

\section{Cas étudiés}

Cette partie est consacrée à la présentation de deux cas d'étude réalisés via l'utilisation de la NFSC. La première étude concerne le rayonnement d'une ligne micro-ruban. Elle permet notamment d'illustrer les phénomènes d'onde stationnaire dans les guides d'onde. La deuxième étude concerne la cartographie d'une boucle à verrouillage de phase hyperfréquence. La cartographie permet notamment de localiser les zones principales de rayonnement du circuit.

\section{$\underline{\text { Rayonnement d'une ligne micro-ruban et ondes stationnaires }}$}

L'étude du rayonnement d'une ligne micro-ruban qui est un élément très bien connu et que l'on retrouve sur la plupart des cartes électroniques permet parfaitement de démontrer les capacités de la NFSC. L'étude permet de plus d'illustrer les phénomènes d'adaptation d'impédance et d'onde stationnaire. La figure 15 (a) présente les lignes de champ théoriques rayonnées par une ligne micro-ruban en zone de champ proche. La figure 15 (a) ainsi que les lignes de champ magnétique tournent autour du ruban tandis que les lignes de champ électrique relient le ruban au plan de masse. En observant ces lignes de champ, on note rapidement par exemple que la composante verticale du champ électrique sera principalement détectée juste au-dessus du ruban (appelée lobe principal) ainsi que sur les bords du ruban (appelée lobes secondaires). La cartographie de la composante verticale du champ électrique rayonnée à $1 \mathrm{~mm}$ au-dessus de la ligne micro-ruban laissée en circuit ouvert est présentée sur la figure 15 (b).

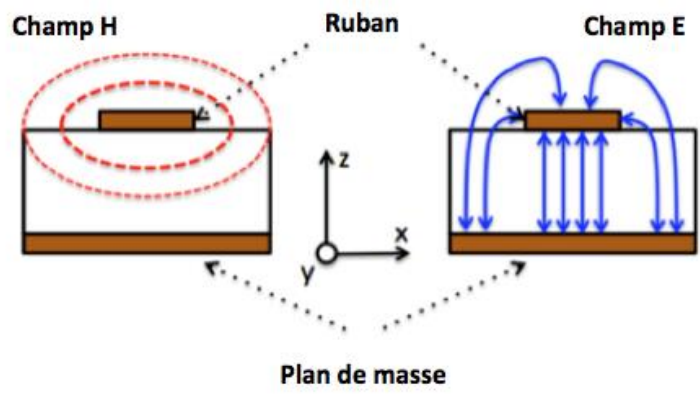

(a)

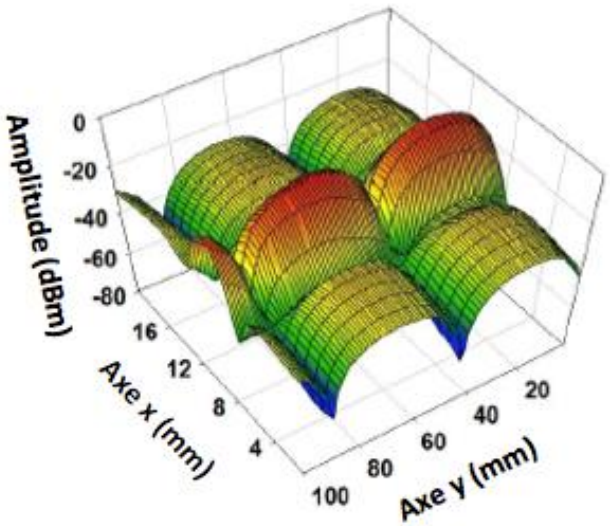

(b)

Fig. 15. (a) Champ électrique et magnétique théorique rayonnés par une ligne micro-ruban, (b) Rayonnement de la composante verticale du champ électrique mesuré à $1 \mathrm{~mm}$ au-dessus de la ligne micro-ruban.

Du fait qu'elle est laissée en circuit ouvert, le rayonnement de la piste présente aussi des ondes stationnaires caractérisées par l'apparition de maximas et minimas de champ électrique correspondant aux différents ventres et nœuds de tension présents sur la ligne. 
En déterminant la distance entre deux maximas, il est alors possible de déduire la longueur d'onde du signal circulant dans la ligne et ainsi de remonter à la valeur de paramètres physiques tels que la permittivité du diélectrique constituant la ligne. En comparant les cartographies du champ électrique et magnétique il est aussi possible de montrer la position spatiale des différents ventres et nœuds de tension et de courant présents sur la ligne, comme illustré par les cartographies des composantes verticale du champ électrique et horizontale du champ magnétique rayonnées par une ligne micro présentées sur la figure 16.

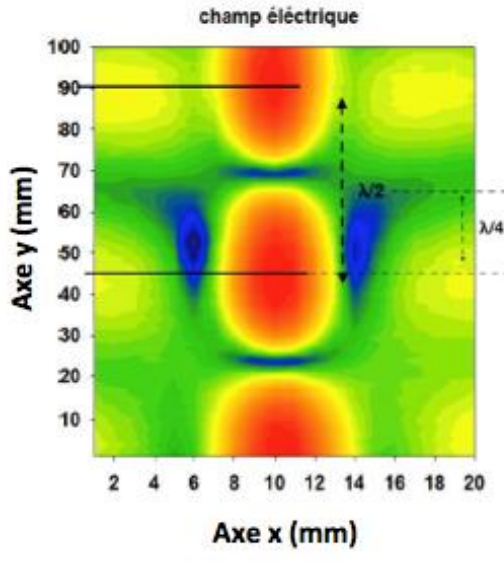

(a)

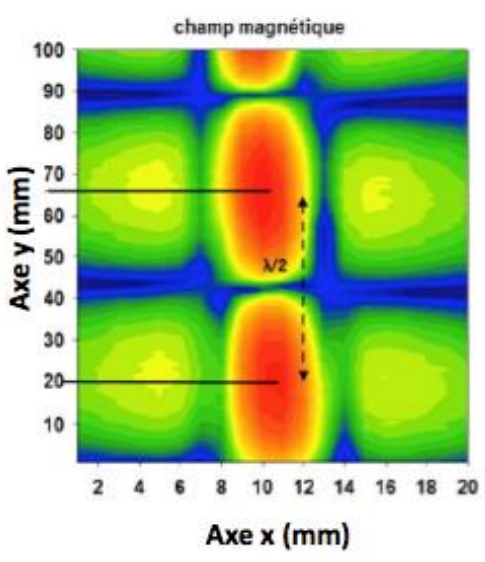

(b)

Fig. 16. Rayonnement de la composante (a) verticale du champ électrique et (b) horizontale du champ magnétique à $1 \mathrm{~mm}$ au-dessus de la ligne micro-ruban. $\lambda$ correspond à la longueur d'onde du signal circulant sur la ligne micro-ruban.

\section{$\underline{\text { Rayonnement d'une boucle à verrouillage de phase }}$}

Une des problématiques de la compatibilité électromagnétique est l'étude du rayonnement de circuits électroniques. Tout circuit en fonctionnement émet des champs électromagnétiques susceptibles de se coupler à des circuits voisins et de les perturber. Le cas d'étude présenté ici concerne l'étude du rayonnement d'une boucle à verrouillage de phase (PLL en anglais) permettant de fournir un signal de fréquence stable de $3040 \mathrm{MHz}$ à partir d'un signal de fréquence égale à $190 \mathrm{MHz}$. La PLL est composée d'un comparateur de phase/fréquence, d'un filtre passe bas et d'un oscillateur contrôlé en tension (VCO en anglais) comme présenté sur la figure 17.

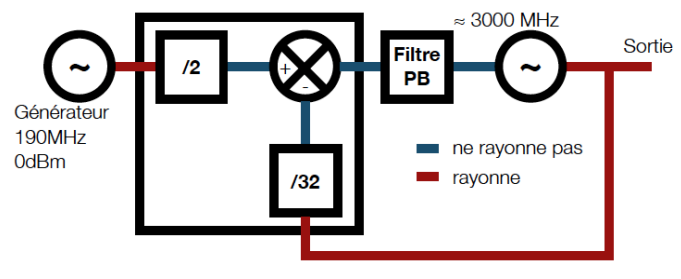

(a)

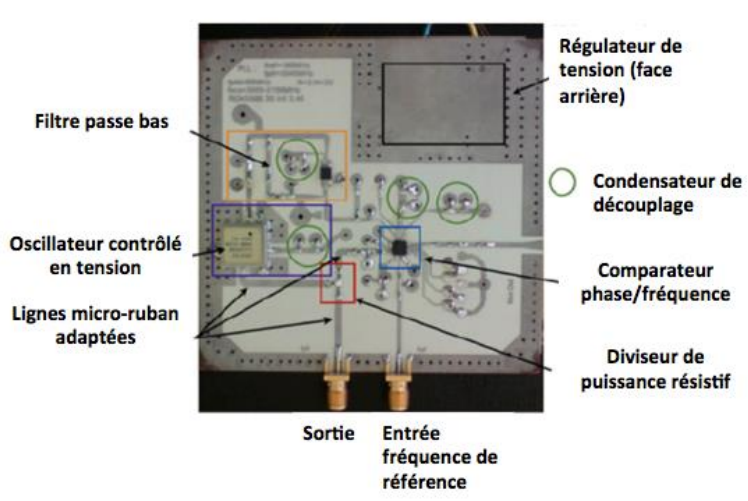

(b)

Fig. 17. (a) Schéma bloc et (b) carte réalisée de la PLL. 
La figure 18 présente le résultat de la cartographie de la composante verticale du champ électrique rayonné à $1 \mathrm{~mm}$ au-dessus de la carte PLL.

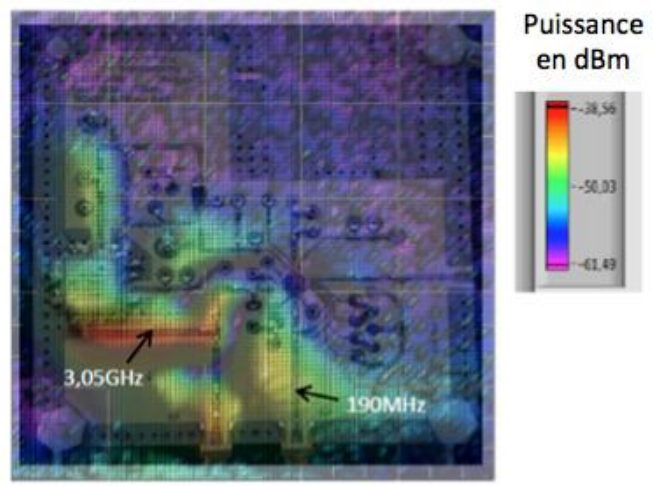

Fig. 18. Résultat de la cartographie de la composante verticale du champ électrique rayonné à $1 \mathrm{~mm}$ audessus de la carte.

Le champ électrique rayonné est principalement localisé au-dessus des pistes sur lesquelles circule un signal de fréquence élevée. En effet, les principales zones de rayonnement sont localisées sur les trois pistes connectées au diviseur résistif sur lesquelles un signal de $3040 \mathrm{MHz}$ circule et sur la piste d'entrée du signal de référence ou un signal de $190 \mathrm{MHz}$ circule.

\section{Conclusions}

Cet article a présenté un outil pédagogique appelé «Mallette Scan Champ Proche ». Cet outil a été développé en se basant sur un banc de mesure utilisé en recherche dans le domaine de la compatibilité électromagnétique des composants et circuits électroniques. Il permet de réaliser des cartographies des différentes composantes du champ électrique et magnétique rayonnées par un circuit électronique. Le projet de construction d'une mallette scan champ proche avait plusieurs buts. Un premier but consistait à faire réaliser le banc de mesure par des étudiants de niveaux variés allant de la L2 au M1 dans le cadre de projets ou de mini-stages abordant différentes thématiques du domaine de l'EEA comme par exemple : l'électronique de puissance, les hyperfréquences, l'informatique industrie... Le deuxième but était de concevoir un outil pédagogique peu couteux (environ $1.000 €$ ) que les enseignants pourraient emporter avec eux pendant les cours afin d'illustrer des phénomènes physiques complexes tels que les phénomènes d'onde stationnaire rencontrés dans le domaine des hyperfréquences ou encore des phénomènes de rayonnement électromagnétique des cartes électroniques rencontrés dans le domaine de la CEM.

Trois points importants du cahier des charges de la mallette ont orienté sa conception. Le premier point était qu'elle soit autonome d'un point de vu énergie, ce qui a été réalisé par l'utilisation d'une batterie et d'une carte d'alimentation spécifique. Le deuxième point était que les résultats soient accessibles facilement à travers une tablette PC ou un smartphone. Ce but a été atteint en utilisant une carte Raspberry-pi permettant d'afficher les résultats de mesure sur une page web accessible depuis n'importe quel appareil équipé de wifi. Enfin le troisième point était de rendre le banc de mesure facilement transportable. Ce dernier point reste à réaliser en intégrant le banc de mesure à une mallette. 


\section{Remerciements}

Nous tenons à remercier les étudiants qui ont participé à la conception de cette mallette scan champ proche : Thomas Chancellier (Stage L2 IMSAT - 1 mois), Ugo D'Aleo (Stage L2 IMSAT - 1 mois), Clément Latry (Projet LPro MMM - 1 mois), Denis Chang (Projet LPro MMM - 100h), Mathieu Maurès (Projet L3 EEA - 1 mois), Quentin Thépault (Projet L3 EEA - 1 mois), Samsul Arefin (M1 EEA) - 3 semaines), Bourama Coulibaly (M1 EEA - 3 semaines), Julian Mejia (M1 EEA - 4 semaines), Gabriel Pelage (M1 EEA) - 4 semaines), Antoine Olivier (M1 EEA - 4 semaines). Ce travail a été réalisé avec le support du GIP-CNFM: Groupement d'Intérêt Public - Coordination Nationale pour la formation en Microélectronique et en nanotechnologies, et le programme d'investissement d'avenir dans le cadre du projet IDEFI-FINMINA : Initiative d'Excellence - Formation Innovante en MIcroélectronique et Nanotechnologies, ANR-11-IDFI-0017

\section{Références}

1. T. Dubois, Etude de l'Effet d'Onde Electromagnétique sur le Fonctionnement de Circuits Electroniques - Mise en Place d'une Méthode de Test des Systèmes, thèse de doctorat, Université de Montpellier (2009).

2. A. Boyer, Méthode de la Prédiction de la Compatibilité Electromagnétique des Systèmes en Boîtier, thèse de doctorat, Université de Toulouse (2007).

3. T. Dubois, G. Duchamp, J. Weckbrodt, S. Azzopardi, Near-Field Scan Tools for Embedded Electronic Analysis, Proceding Conférence PANPAC 2016 (2016).

4. D. Baudry, A. Louis, B. Mazari, "Characterization of the Open-Ended Coaxial Probe Used for NearField Measurements in EMC Applications”, Progress In Electromagnetics Research, pp. 311-333, 2006.

5. S. Jarrix, T. Dubois, R. Adam, P. Nouvel, B. Azais, D. Gasquet, Probe Characterization for Electromagnetic Near-Field Studies, IEEE Trans. On I\&M (2008). 